\title{
Tracking and Stabilization of Chaotic System with Static Uncertain Nonlinear Functions
}

\author{
${ }^{1, \mathrm{a}}$ Zhanlei Shang, ${ }^{1, \mathrm{~b}}$ Yibing Han, ${ }^{2, \mathrm{c}}$ Ruiqi Wang and ${ }^{3, \mathrm{~d}}$ Junwei Lei \\ ${ }^{1}$ College of Computer and Communication Engineering, Zhengzhou University of \\ Light Industry, Zhengzhou 450002, China \\ ${ }^{2}$ Department of Armament Engineering, Naval Aeronautical and Astronautical \\ University, Yantai Shandong 264001, China \\ ${ }^{3}$ Department of Control Engineering, Naval Aeronautical and Astronautical \\ University, Yantai Shandong 264001, China

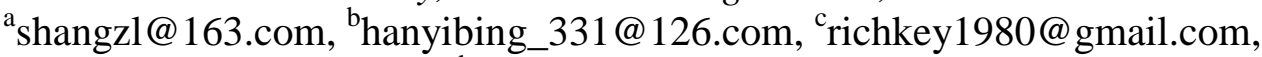 \\ dleijunwei@126.com,
}

\begin{abstract}
Tracking and stabilization are two typical problems of chaotic system control. A unit error model is built for the above both two problems and a kind of adaptive control is designed to solve a special kind of system uncertainty. The uncertain nonlinear function. Is not required to be bounded compared with a known smooth positive function. Also a kind of approximation method is proposed for nonlinear functions based on Fourier series method. At last, a four dimension chaotic system is taken as an example to do the numerical simulation to testify the rightness of the proposed method.
\end{abstract}

Keywords: tracking, chaotic system, stabilization, error model, nonlinear function, adaptive control

\section{Introduction}

Adaptive control of chaotic systems with uncertain parameters were widely researched in recent years. But the research on chaotic systems with nonlinear functions was only appeared in the past several years. The static nonlinear functions are assumed to satisfy the increasing condition in some papers, or they are assume to the satisfy the so called matched condition in other papers. This condition was relaxed in the following research paper [1] and [2]. And paper [3] required both the bound function and bound coefficient of static uncertain function should be known. And paper [3] was mainly focus on the situation of multi-dimension chaotic system with single uncertain nonlinear function. Also the result of above research can only be applied in unit chaotic system with same structures. So there are some research work should be added if the same method is applied in control a general chaotic system ${ }^{[1-3]}$.

In this paper, a kind of general chaotic system model with static nonlinear functions is taken as an example and a kind of adaptive control law is designed to solve the tracking and stabilization problem of chaotic systems. Compared with paper [3], the requirement for nonlinear function is fatherly relaxed. In paper [1-3], the bound function and bound of nonlinear function are need to be known, but in this paper, the bound function is required to be exist and only one part of it is need to be known ${ }^{[4-6]}$.

\section{Problem Description}

Consider the typical chaotic system with uncertain nonlinear functions as follows:

$$
\dot{x}=f(x)+\Delta(x)+b u
$$


Where $x=\left[x_{1}, \cdots, x_{n}\right]^{T}, u=\left[u_{1}, \cdots, u_{n}\right]^{T}$ are vector and their dimension are $n$.

Take a four dimension system as an example, it can be written as:

$$
\begin{gathered}
\dot{x}_{1}=f_{1}\left(x_{1}, \cdots, x_{4}\right)+\Delta_{1}\left(x_{1}, \cdots, x_{4}\right)+b_{1} u_{1} \\
\dot{x}_{2}=f_{2}\left(x_{1}, \cdots, x_{4}\right)+\Delta_{2}\left(x_{1}, \cdots, x_{4}\right)+b_{2} u_{2} \\
\dot{x}_{3}=f_{3}\left(x_{1}, \cdots, x_{4}\right)+\Delta_{3}\left(x_{1}, \cdots, x_{4}\right)+b_{3} u_{3} \\
\dot{x}_{4}=f_{4}\left(x_{1}, \cdots, x_{4}\right)+\Delta_{4}\left(x_{1}, \cdots, x_{4}\right)+b_{4} u_{4}
\end{gathered}
$$

Where $f(x)$ is a known function of system, and $\Delta(x)$ is the description of unknown part of system, and it is assumed to be described by static nonlinear functions. And $b_{i}$ is known constant. To make it easy to understand, the unknown parts of the system are assumed to be described by static nonlinear function ${ }^{[7-9]}$.

The above model is typical and most of well known chaotic systems can be written as above format, such as Lorenz chaotic system

$$
\begin{aligned}
& \dot{x}_{1}=\alpha_{1}\left(y_{1}-x_{1}\right) \\
& \dot{y}_{1}=\gamma_{1} x_{1}-x_{1} z_{1}-y_{1} \\
& \dot{z}_{1}=x_{1} y_{1}-\beta_{1} z_{1}
\end{aligned}
$$

The objective of stabilization problem of chaotic system is to design a control law $u=u(x, \hat{\theta}, \hat{q}), \dot{\hat{\theta}}=g(x, \hat{\theta}), \dot{\hat{q}}=g(x, \hat{q})$ such that the state of system can converge to zero, it means that $x \rightarrow 0$.

The objective of tracking problem of chaotic system is to design a control law $u=u(x, \hat{\theta}, \hat{q}), \dot{\hat{\theta}}=g(x, \hat{\theta}), \dot{\hat{q}}=g(x, \hat{q})$ such that the state of system can track the desired value, it means that $x \rightarrow x^{d}$.

Assume the desired value is $x_{i}^{d}$, and it is a constant so $\dot{x}_{i}^{d}=0$, then the stabilization problem can be a special situation of tracking problem that $x_{i}^{d}=0$.

Define $z_{i}=x_{i}-x_{i}^{d}$, then the stabilization problem and tracking problem can use a unit error model to describe as follows ${ }^{[10-13]}$.

$$
\dot{z}_{i}=f_{i}\left(x_{1}, \cdots, x_{4}\right)+\Delta_{i}\left(x_{1}, \cdots, x_{4}\right)+b_{i} u_{i}
$$

\section{Assumption}

Several assumptions are proposed as below to simplify the analysis the problem.

Assumption 1: for $1 \leq i \leq n$, there exists a unknown positive constant $q_{i}^{*} \leq d_{i}$ such that

$$
\left|\Delta_{i}(X, t)\right| \leq q_{i}^{*} \psi_{i}(X)
$$

where $d_{i}$ is a known constant and $\psi_{i}(X)$ is a known positive smooth function.

Consider that it is difficult of get $\psi_{i}(X)$ of a real system, a kind of simple situation that $X$ is a scalar is research first . 
Assumption 2: $\Delta_{i}(X, t)$ satisfies the Fourier condition to be expanded so there exists a Fourier condition that

$$
\Delta_{i}(X, t) \approx \sum_{i=0}^{n} a_{i j} \sin i j x+b_{i j} \cos i j x
$$

Assumption 3: $\Delta_{i}(X, t)$ satisfies the absolute integrable condition, then it holds:

$\lim _{j \rightarrow \infty} a_{i j} \rightarrow 0 \quad \lim _{j \rightarrow \infty} b_{j} \rightarrow 0$

So there exists $\varepsilon_{i}>0$ for $N$ satisfies:

$$
\left|\Delta_{i}(X, t)-\left(\sum_{j=0}^{N} a_{i j} \sin j x+b_{i j} \cos j x\right)\right|<\varepsilon_{i}
$$

Then it can be written as

$$
\begin{aligned}
& \Delta_{i}(X, t)<\varepsilon_{i}+\left|\sum_{j=0}^{N}\left(a_{i j} \sin j x+b_{i j} \cos j x\right)\right| \\
& \leq \varepsilon_{i}+\left|\sum_{j=0}^{N}\left(a_{i j} \sin j x\right)\right|+\left|\sum_{j=0}^{N}\left(b_{i j} \cos j x\right)\right| \\
& \leq \varepsilon_{i}+\sum_{j=0}^{N}\left(a_{i j}|\sin j x|\right)+\sum_{j=0}^{N}\left(b_{i j}|\cos j x|\right)
\end{aligned}
$$

Assumption $4:$ choose $\psi_{i}(X)=\sum_{j=0}^{N}|\sin j x|+\sum_{j=0}^{N}|\cos j x|+1$ and $q_{i}^{*}=\max \left(a_{i}, b_{i}, \frac{\varepsilon_{i}}{\max \left(a_{i}, b_{i}\right)}\right)$ is an unknown constant ${ }^{[14-15]}$.

\section{The Design of Robust Adaptive Controller}

Considering the $i^{\text {th }}$ subsystems as below

$$
\dot{z}_{i}=f_{i}\left(x_{1}, \cdots, x_{4}\right)+\Delta_{i}\left(x_{1}, \cdots, x_{4}\right)+b_{i} u_{i}
$$

Then design of control $u_{i}$ is as follows:

$$
\begin{gathered}
u_{i}=f_{2 i}(x)\left[-f_{i}\left(x_{1}, \cdots, x_{4}\right)-\hat{q}_{i}^{*} \psi_{i}(x) \operatorname{sign}\left(z_{i}\right)-f_{z i}\left(z_{i}\right)\right] \\
f_{2 i}(x)=b_{i}^{-1} \\
f_{z i}\left(z_{i}\right)=k_{i 1} z_{i}+k_{i 2} \frac{z_{i}}{\left|z_{i}\right|+\varepsilon_{i 1}}+k_{i 3} \frac{3}{2} z_{i}^{1 / 3} \exp \left(z_{i}^{2 / 3}\right)+k_{i 4} \operatorname{sign}\left(z_{i}\right)
\end{gathered}
$$

Then

$$
\begin{aligned}
& z_{i} \dot{z}_{i}=z_{i}\left[\Delta_{i}(x)-\hat{q}_{i}^{*} \psi_{i}(x) \operatorname{sign}\left(z_{i}\right)-f_{z i}\left(z_{i}\right)\right] \leq-z_{i} f_{z i}\left(z_{i}\right) \\
& +\left|z_{i}\right| q_{i}^{*} \psi_{i}(x)-\hat{q}_{i}^{*} \psi_{i}(x)\left|z_{i}\right|=-z_{i} f_{z i}\left(z_{i}\right)+\left|z_{i}\right| \tilde{q}_{i}^{*} \psi_{i}(x)
\end{aligned}
$$

Define a new variable $\tilde{q}_{i}$ as : $\tilde{q}_{i}=q_{i}^{*}-\hat{q}_{i}$, then: $\dot{\tilde{q}}_{i}=-\dot{\hat{q}}_{i}=\psi_{i}(x)\left|z_{i}\right|$. 
Note 1:To eliminate the discrete switching of sign function, a kind of soft function is adopted to take place of the sign function. Then the control law can be revised as:

$$
u_{i}=f_{2 i}(x)\left[-f_{i}\left(x_{1}, \cdots, x_{4}\right)-\hat{q}_{i}^{*} \psi_{i}(x) \frac{z_{i}}{\left|z_{i}\right|+\varepsilon_{b i}}-f_{z i}\left(z_{i}\right)\right]
$$

Then it can rewritten as

$$
\begin{aligned}
& z_{i} \dot{z}_{i}=z_{i}\left[\Delta_{i}(x)-\hat{q}_{i}^{*} \psi_{i}(x) \operatorname{sign}\left(z_{i}\right)-f_{z i}\left(z_{i}\right)\right] \leq-z_{i} f_{z i}\left(z_{i}\right)+\left|z_{i}\right| q_{i}^{*} \psi_{i}(x) \\
& -\hat{q}_{i}^{*} \psi_{i}(x) \frac{\left|z_{i}\right|^{2}}{\left|z_{i}\right|+\varepsilon_{b i}}=-z_{i} f_{z i}\left(z_{i}\right)+\left|z_{i}\right| \tilde{q}_{i}^{*} \psi_{i}(x)
\end{aligned}
$$

Define $\tilde{q}_{i}=q_{i}^{*}-\hat{q}_{i} \frac{\left|z_{i}\right|}{\left|z_{i}\right|+\varepsilon_{b i}}$, when $\left|z_{i}\right| \square \varepsilon_{b i}, \tilde{q}_{i} \square q_{i}^{*}-\hat{q}_{i}$, Then $\dot{\tilde{q}}_{i}=-\dot{\hat{q}}_{i}=\psi_{i}(x)\left|z_{i}\right|$. The system can be guaranteed to converge to small neighborhood of $\left|z_{i}\right| \leq \varepsilon_{b i}$.

Note 2: There is also another method to eliminate the discontinuous switching of sign function. It is that the sign function can be removed directly, then the control law can be designed as follows:

$$
u_{i}=f_{2 i}(x)\left[-f_{i}\left(x_{1}, \cdots, x_{4}\right)-\hat{q}_{i}^{*} \psi_{i}(x)-f_{z i}\left(z_{i}\right)\right]
$$

Then it holds

$$
\begin{aligned}
& z_{i} \dot{z}_{i}=z_{i}\left[\Delta_{i}(x)-\hat{q}_{i}^{*} \psi_{i}(x) \operatorname{sign}\left(z_{i}\right)-f_{z i}\left(z_{i}\right)\right] \leq-z_{i} f_{z i}\left(z_{i}\right) \\
& +\left|z_{i}\right| q_{i}^{*} \psi_{i}(x)-\hat{q}_{i}^{*} \psi_{i}(x) z_{i}=-z_{i} f_{z i}\left(z_{i}\right)+\left|z_{i}\right| \tilde{q}_{i}^{*} \psi_{i}(x)
\end{aligned}
$$

And define

$$
\begin{aligned}
& \tilde{q}_{i}=q_{i}^{*}-\hat{q}_{i} \operatorname{sign}\left(z_{i}\right) \\
& \dot{\tilde{q}}_{i}=-\dot{\hat{q}}_{i} \operatorname{sign}\left(z_{i}\right)
\end{aligned}
$$

Selecte $\dot{\hat{q}}_{i}=\operatorname{sign}\left(z_{i}\right) \psi_{i}(x)\left|z_{i}\right|$, it is easy to prove the system is also stable with this adaptive turning law ${ }^{[16-17]}$. Compare the above several methods, the last selection of control law is reasonable.

\section{The Analysis of Unknown Nonlinear Function Approximation}

Note 3: If the structural information of unknown nonlinear is known, then assume that

$$
\left|\Delta_{i}(X, t)\right| \leq \sum_{j=1}^{n} c_{j i} \psi_{j i}(x)
$$

where $c_{i}$ is the unknown parameter and $\psi_{i}(x)$ is the nonlinear function described by the known system information[14-17].

At this time, the control law can be designed as follows:

$$
u_{i}=f_{2 i}(x)\left[-f_{i}\left(x_{1}, \cdots, x_{4}\right)-\sum_{j=1}^{n} \hat{c}_{j i} \psi_{j i}(x)-f_{z i}\left(z_{i}\right)\right]
$$




$$
\dot{\hat{c}}_{j i}=\operatorname{sign}\left(z_{i}\right) \psi_{j i}(x)\left|z_{i}\right|
$$

It is easy to prove the design is reasonable.

Note 4: if it is difficult to find a known function as $\psi_{i}(x)$, then we consider one of the simplest situation as below. Assume it satisfies

$$
\left|\Delta_{i}(X, t)\right| \leq c_{i}
$$

for the interval $|X|<a_{x}$, then the control laws of system can be designed as follow:

$$
\begin{gathered}
u_{i}=f_{2 i}(x)\left[-f_{i}\left(x_{1}, \cdots, x_{4}\right)-\hat{c}_{i}-f_{z i}\left(z_{i}\right)\right] \\
\dot{\hat{c}}_{i}=z_{i}
\end{gathered}
$$

The control law can be arranged as follow:

$$
u_{i}=f_{2 i}(x)\left[-f_{i}\left(x_{1}, \cdots, x_{4}\right)-\int z_{i} d t-f_{z i}\left(z_{i}\right)\right]
$$

If high gain feedback can be adopted, in the initial period

$$
z_{i} \dot{z}_{i}=z_{i}\left[\Delta_{i}(x)-\int z_{i} d t-f_{z i}\left(z_{i}\right)\right]>0
$$

$z_{i}$ will increase, then it will lead the state over the range $|X|<a_{x}$, then assumptions conditions $\left|\Delta_{i}(X, t)\right| \leq c_{i}$ can not be satisfied, then the system will be unstable.

So there are two main important factors as follows by which the stability of system was mainly affected.

First, try to use a high gain feedback to design the part of $f_{z i}\left(z_{i}\right)$, and the gain can be as big as only if the actual system allows.

Second, try to choose a proper function to describe the system uncertainty such that the interval in above hypothesis is big enough.

Note 5: Considering the following nonlinear function

$$
\left|\Delta_{i}(X, t)\right|=3+4 x
$$

If the interval in above hypothesis is selected as

$$
\left|\Delta_{i}(X, t)\right| \leq d_{i}
$$

The control law is designed as follows.

$$
u_{i}=f_{2 i}(x)\left[-f_{i}\left(x_{1}, \cdots, x_{4}\right)-\int z_{i} d t-f_{z i}\left(z_{i}\right)\right]
$$

Then the assumption conditions come into existence only inside $x \leq\left(d_{i}-3\right) / 4$ for any arbitrary selection of $d_{i}$.

If the hypothesis is selected as

$$
\left|\Delta_{i}(X, t)\right| \leq c_{i}+k_{i}|x|
$$

The control law is designed as follows

$$
u_{i}=f_{2 i}(x)\left[-f_{i}\left(x_{1}, \cdots, x_{4}\right)-\int z_{i} d t-|x| \int z_{i}|x| d t-f_{z i}\left(z_{i}\right)\right]
$$


The parameters can be selected as $c_{i}=3, k_{i}=4$ to make the system hypothesis set up in the global scope. Therefore, the control law is global stable at this time.

It is difficult to know the uncertain structure of actual system in advance. For example:

$$
\left|\Delta_{i}(X, t)\right|=3+4 x^{2}
$$

Then in view of the hypothesis $\left|\Delta_{i}(X, t)\right| \leq d_{i}$, the interval for state $x$ can be solved as

$$
x \leq \sqrt{\left(d_{i}-3\right) / 4}
$$

In view of the hypothesis $\left|\Delta_{i}(X, t)\right| \leq c_{i}+k_{i}|x|$, the interval for state $x$ can be solved as

$$
x \leq k_{i} / 4
$$

Based on the above discussion, it is easy to make a conclusion as follows: if a polynomial is used to approximate an unknown nonlinear function, High order Taylor expansion method can improve the approximation accuracy. However, if the order of polynomial is higher, a bigger feedback gain should be designed for the system. For an actual system, it is easy to enter the saturation area. Also high-gain feedback is also not allowed by a lot of actual systems at the same time. Therefore, there is an irreconcilable contradiction in view of the control problem of uncertain systems.

\section{The Analysis of Numerical Simulation}

Taking the following 4-D hyper-chaotic system as an experiment object, the model can be written as follows.

$$
\begin{gathered}
\dot{x}_{1}=a\left(x_{2}-x_{1}\right)+k_{l b} x_{4} \cos x_{2}+u_{1} \\
\dot{x}_{2}=b x_{1}-k_{1} x_{1} x_{3}+k_{l b}\left(1+\sin \left(x_{2} x_{3}\right)\right) x_{2}+u_{2} \\
\dot{x}_{3}=-c x_{3}+h x_{1}^{2}+k_{l b}\left(2-\cos \left(x_{1} x_{2} x_{3} x_{4}\right)\right) x_{1}+u_{3} \\
\dot{x}_{4}=-d x_{1}+k_{l b} x_{3}\left(3+\sin \left(x_{1} x_{3}\right)\right)+u_{4}
\end{gathered}
$$

Considering unknown nonlinear functions which satisfy the following assumption:

$$
\begin{gathered}
\left|k_{l b} x_{4} \cos x_{2}\right| \leq q_{1}^{*}\left|x_{4}\right|, \quad\left|k_{l b}\left(1+\sin \left(x_{2} x_{3}\right)\right) x_{2}\right| \leq q_{2}^{*}\left|x_{2}\right| \\
\left|k_{l b}\left(2-\cos \left(x_{1} x_{2} x_{3} x_{4}\right)\right) x_{1}\right| \leq q_{3}^{*}\left|x_{1}\right| \\
\left|k_{l b} x_{3}\left(3+\sin \left(x_{1} x_{3}\right)\right)\right| \leq q_{4}^{*}\left|x_{3}\right|
\end{gathered}
$$

when system parameters are chosen as $a=10, b=40, c=2.5, d=10.6$, $k=1, h=4, \quad k_{l b}=0.05$, the system is chaotic. The initial value are $x_{1}(0)=1$, $x_{2}(0)=-1, \quad x_{3}(0)=-2, \quad x_{4}(0)=2$, The simulation results of chaotic free movement trajectories are shown as figure 1 and figure 2 . 


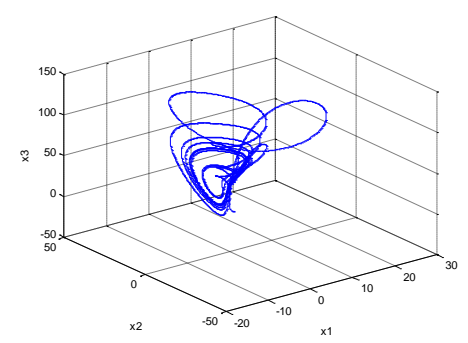

Figure 1. Trajectory of Uncontrolled Chaotic System (1)

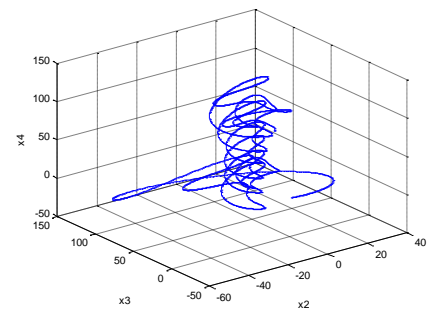

Figure 2. Trajectory of Uncontrolled Chaotic System (2)

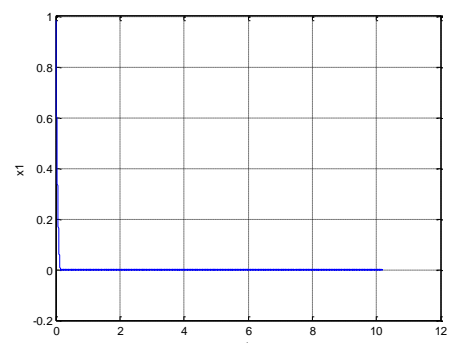

Figure 3. Trajectory of State $\mathrm{X}$

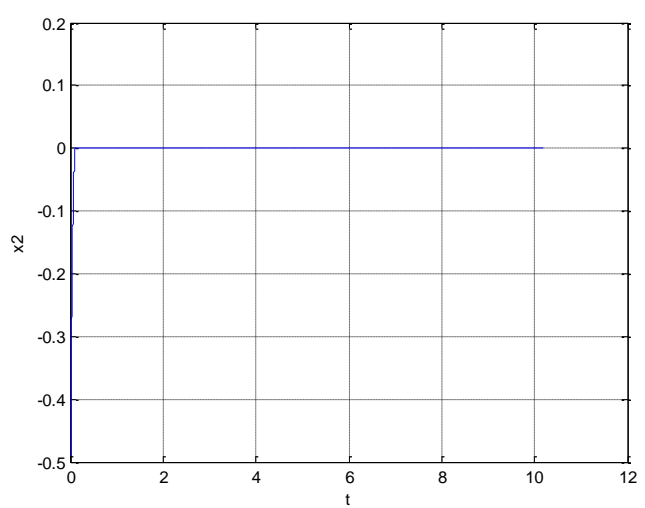

Figure 4. Trajectory of State $Y$ 


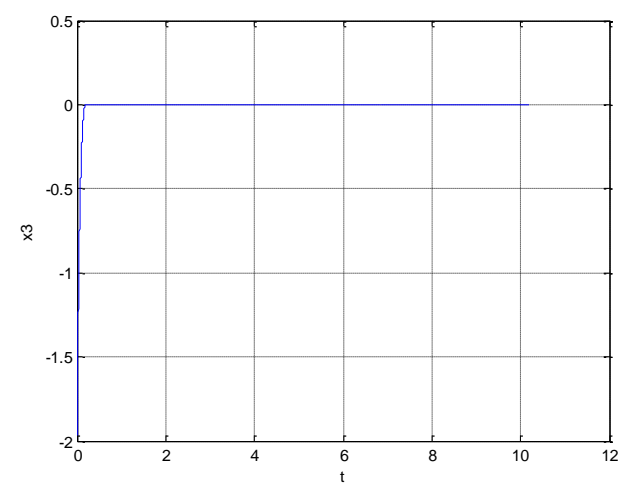

Figure 5. Trajectory of State X3

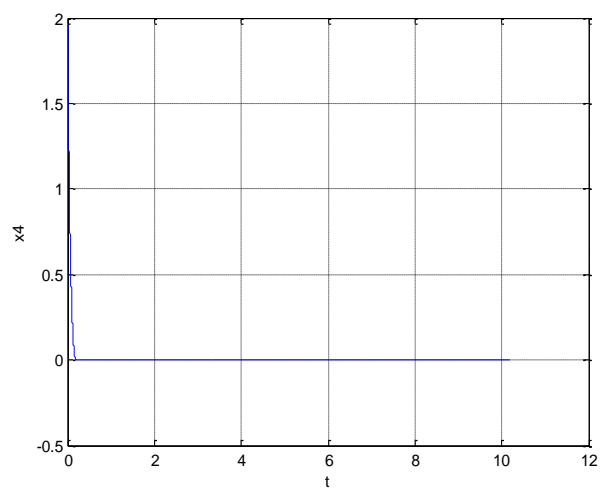

Figure 6. Trajectory of State $\times 4$

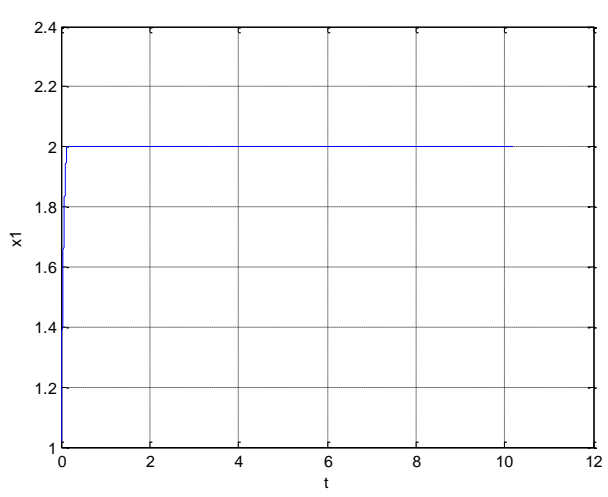

Figure 7. Tracking of State X1 


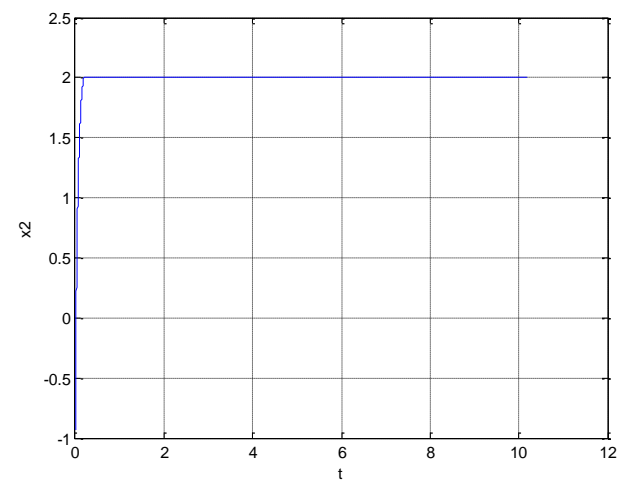

Figure 8 Tracking of State X2

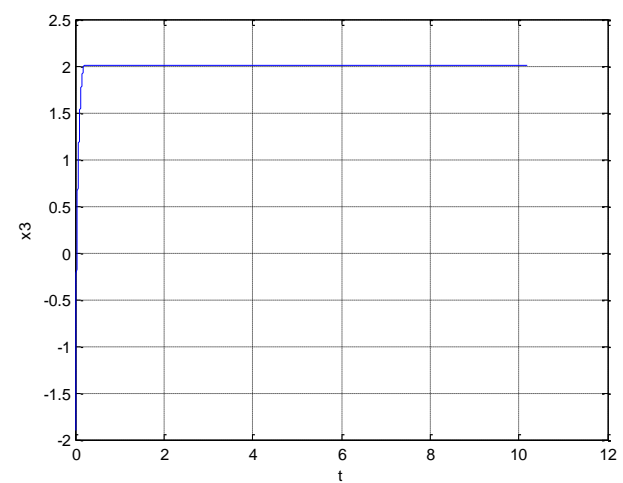

Figure 9. Tracking of State X3

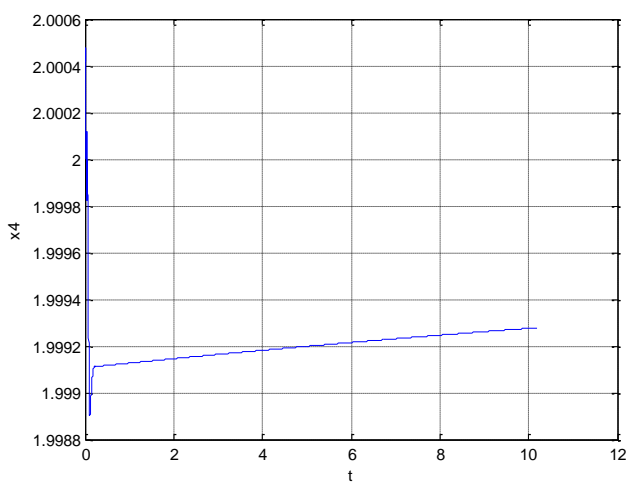

Figure 10. Tracking of State X4

The stabilization process of chaotic system states are shown by figure 3 to figure 6 . And choose an expect value of desired trajectory as 2, the system states tracking process of desired trajectory were shown by figure 7 to figure 10 . The conclusion can be made from simulation results that the chaotic system can realize stabilization and tracking with the proposed method in the existence of static uncertain functions. 


\section{Conclusions}

A kind of robust adaptive strategy was proposed to solve the tracking and stabilization problem of chaotic systems with static nonlinear functions. Especially, several kinds of nonlinear situation of uncertain functions were discussed. Different estimation or approximation methods were used and also different accuracy of approximation was achieved. At last, detailed numerical simulation was done to show the effectiveness of the proposed method.

\section{References}

[1] GE S.S. and Wang C, "Adaptive control of uncertain Chua's circuits", IEEE Trans Circuits System., vol. 47 no. 9, (2000), pp.1397-1402.

[2] Pecora L M and Carroll T L., "Synchronization in chaotic systems". Phys.Rev. Lett, vol. 64: (1990), pp. 821-82.

[3] C. Tsung-Ying, L. Jui-Sheng, L. Teh-Lu and Y., Jun-Juh "Antisynchronization of uncertain unified chaotic systems with dead-zone nonlinearity”, Nonlinear Analysis 68 (2008), pp. 2629-2637, Physics Letters A 350, (2006), pp. 36-43.

[4] Alexander L., Fradkov, and Markov A. Yu, "Adaptive synchronization of chaotic systems based on speed gradient method and passification”, IEEE Trans Circuits System, vol. 44, no. 10, (1997), pp. 905912.

[5] C. Liang, C. Guanrong, and Y.W. Lee, "Fuzzy modeling and adaptive control of uncertain chaotic systems[J]", Information Sciences, vol. 121, no. 1, (1999), pp. 27-37.

[6] C. Liang and C. Guanrong, "Fuzzy presictive control of uncertain chaotic Systems using time series", Int. J Bifurcation and chaos. vol. 9, no. 40, (1999), pp. 757-767.

[7] Y. Jianping and L. Changpin, "On synchronization of three chaotic systems", Chaos,Solitons and fracrals, no. 23, (2005), pp. 1683-1688.

[8] C. Sarasola, F.J.Torrealdea and A.D. Anjou, "Feedback synchronization of chaotic Systems" Int.J.Bifurcation and chaos. vol. 13, no. 1, (2003), pp. 177-191.

[9] H. Ming-chung and H. Yao-Chen. "Synchronization of two different systems by Using generalized active control", Physics Letters A. no. 301, (2002), pp. 424-428.

[10] E.M. Elabbasy, H.N. Agiza and M.M. El-dessoky, "Adaptive synchronization of lv System with uncertain parameters", Chaos,solitons and fractals, no. 21, (2004), pp. 657-667.

[11] C.C. Fuh and P.C Tung, "Controlling chaos using differential geometric method", Phys. Rev Lett; vol. 75, (1995), pp. 2952-5.

[12] Chen G, Dong X. "On feedback control of chaotic continuous-time systems", IEEE Trans Circ Syst; vol. 40, (1993), pp. 591-601.

[13] X. Wu and J. Lu, "Parameter identification and backs tepping control of uncertain Lv system", Chaos, Solitons \& Fractals; vol. 18, (2003), pp. 721-9.

[14] T. Fang and W. Ling, "An adaptive active control for the modified Chua's circuit, Physics Letters A, vol. 346, (2005), pp.342-346.

[15] H. Manfeng, X. Zhenyuan, Z. Rong and H. Aihua, "Parameters identification and adaptive full state hybrid projective synchronization of chaotic (hyper-chaotic) systems, Physics Letters A", vol. 361, (2007), pp.231-237.

[16] J.W. Lei and Y. Wang, "Terminal sliding mode control of unit choatic system", Journal of Naval Aeronautical and Astronautical University, vol. 22, no. 3, (2007), pp. 309-312.

[17] H.K. Chen, "Global chaos synchronization of new chaotic systems via nonlinear control", Chaos, Solitons, Fractals, vol. 23, (2005), pp. 1245-1251. 